\title{
ADDISONIAN CRISIS PRECIPITATED BY THYROXINE THERAPY IN A PATIENT WITH TYPE 2 AUTOIMMUNE POLYGLANDULAR SYNDROME
}

\author{
S Pokharel ${ }^{1 *}$, A Shrestha ${ }^{1}$, D Maskey ${ }^{1}$, B Shrestha ${ }^{1}$, P Paudel ${ }^{1}$, B Shrestha ${ }^{1}$, BM Dhital ${ }^{1}$ \\ ${ }^{1}$ Department of Medicine, Chitwan Medical College, Bharatpur, Chitwan, Nepal. \\ *Correspondence to : Dr Sunil Pokharel, Department of Medicine, Chitwan Medical College, Bharatpur, Chitwan Nepal. \\ Email: sunilpokharel786@hotmail.com
}

\begin{abstract}
Primary hypoadrenalism(Addison's disease) refers to glucocorticoid deficiency occurring in the setting of adrenal disease(mostly due to autoimmune adrenalitis), whereas secondary hypoadrenalism arises because of deficiency of $\mathrm{ACTH}(\mathrm{mostly}$ due to pituitary disease). A major distinction between these two is that mineralocorticoid deficiency invariably accompanies primary hypoadrenalism, but this does not occur in secondary hypoadrenalism because only ACTH is deficient; the renin-angiotensin-aldosterone axis is intact and in primary hypoadrenalism skin pigmentation is always present due to increased ACTH secretion(unless of short duration) but it is absent in secondary hypoadrenalism. Addison's disease or primary adrenocortical failure was first described by English physician Thomas Addison, who found it in six patients with adrenal tuberculosis in 1855(1). Addisonian crisis is a potentially fatal condition associated mainly with an acute deficiency of the glucocorticoid cortisol and, to a lesser extent, the mineralocorticoid aldosterone. This is a rare condition with an estimated incidence in the developed world of 0.8 cases per 100,000 and prevalence of 4 to 11 cases/100,000 population. Despite optimised life-saving glucocorticoid replacement and mineralocorticoid-replacement therapy, it is life threatening if overlooked(2-3). Hypothyroidism may mask the addison's disease so in patients with panhypopituitarism and autoimmune polyglandular syndrome type 2 thyroxine replacement without adequate steroid replacement may precipitate acute addisonian crisis.
\end{abstract}

Key Words: Addison's disease, Autoimmune polyglandular syndrome Type-2, Hypoadrenalism, Hypothyroidism, Thyroxine.

\section{INTRODUCTION}

In the western world, autoimmune adrenalitis accounts for over $70 \%$ cases of addison's disease. ${ }^{4}$ Fifty percent of patients with this form of addison's disease have an associated autoimmune disease, thyroid disease being the most common. ${ }^{4}$ The original description by Schmidt in 1926 of autoimmune disease affecting more than one organ concerned a patient with thyroiditis and hypoadrenalism. ${ }^{5,6}$ The syndrome is now defined as autoimmune adrenal disease associated with autoimmune thyroid disease and/or type 1 diabetes and is referred to as APS2. The most common combination (around 75\% of cases) is of thyroid and adrenal failure, and either organ may fail first. ${ }^{7}$ In those patients who have a combination of type 1 diabetes and adrenal failure, the diabetes typically occurs first ${ }^{8}$ and an unexpected fall in insulin requirements may be the earliest indication of impending adrenal failure. ${ }^{9}$ About $10 \%$ of cases have all three of the major conditions $^{7}$ (type 1 diabetes mellitus plus autoimmune adrenal and thyroid disease). Autoimmune polyglandular syndrome type 2, a form of autoimmune polyendocrine syndrome also known as Schmidt's syndrome ${ }^{10}$ or APS-II, is the most common form of the polyglandular failure syndromes. ${ }^{11}$ A fraction of the patients also present with or later develop other organ specific autoimmune disorders. These include hypergonodotropic hypogonadism, vitiligo, chronic atrophic gastritis, pernicious anaemia, autoimmune chronic hepatitis and celiac disease. Many circulating organ-specific antibodies directed against endocrine organs are detected in patients with APS II. Major relevant autoantibodies detectable at the clinical onset of the diseases constituting type II APS are-21 hydroxylase autoantibodies (21OHAb) against adrenal cortex, thyroperoxidase (TPOAb), thyroglobulin (TGAb) and TSH receptor autoantibodies (TRAb) against thyroid; insulin (IAA), and glutamic acid decarboxylase autoantibodies (GADAb) against the endocrine pancreas. ${ }^{12}$ The destructive process in APS2 is believed to be a cell-mediated immune response and a loss of self-tolerance . Although the genetic basis of the condition has not been clearly defined, it is strongly linked to various alleles within the HLADR3-carrying haplotype or related genes. ${ }^{13}$ As HLA molecules largely determine T-cell responses to antigens, both an external antigen stimulus and a genetic susceptibility may be required to initiate the autoimmune destructive process. The wide range of endocrine-related autoantibodies reported in APS2 indicates that $\mathrm{B}$ cells are activated and contribute to the pathologic process $^{14}$ Extensive tissue destruction must occur before the process becomes clinically apparent; one report estimated that 
80 to $90 \%$ of adrenal tissue must be destroyed before symptoms of Addison's disease occur. ${ }^{15}$ It is more heterogeneous and has not been linked to one gene. Rather, patients are at a higher risk when they carry a particular human leukocyte antigen genotype (DQ2, DQ8 and DRB1*0404). APS-II affects women to a greater degree than men ( $75 \%$ of cases occur in women). ${ }^{8}$

\section{CASE PRESENTATION}

A 35 years old male patient of brahmin cast was admitted in our hospital with chief complaint of anorexia, multiple episodes of vomiting and loose motion which was watery in nature with no mucus and blood present in his stool for 2 days ,without associated fever, pain abdomen .On further enquiry about the history the patient admitted that his skin was fair in colour few years back and without any obvious region his skin started to become dark, he also started to feel unusually tired, lethargic and lost his appetite for food. He was working as a manager (indoor job) in Quatar 2 years ago, but as he was feeling exceedingly tired and lethargic he left his job and came back to Nepal and was staying at home since last 1year. The history was confirmed by comparision of his photographs taken few years back where his complexion was fair. Patient also gives history of having diagnosed as overt hypothyroidism 3 months back with values -(FT3:3.77pg/ml(2.0-4.4)FT4:0.57ng/ $\mathrm{dl}(0.93-1.70) \mathrm{TSH}: 30.43 \mathrm{microIU} / \mathrm{ml}(0.27-4.20))$ and started on Thyroxine replacement therapy. The patient also gave a similar past history of lethargy, decreased appetite and 2 episodes of diarrhea in the previous month for which he was admitted in local hospital and was treated accordingly.He also gave history of significant weight loss in this 2 years (around $15 \mathrm{~kg}$ ). He has no known history of pulmonary tuberculosis or a family history of such illness.

\section{On admission :}

Examination: Vitals-blood pressure was SBP:70 mmof $\mathrm{Hg}$ (Palpatory)Pulse:120bpm (feeble).

Skin- hyperpigmented (pigmentation in his bilateral palms, buccal cavity and tongue)

\section{Lab reports as follows:}

$\mathrm{Na}+: 138.2 \mathrm{mg} / \mathrm{dl}, \quad \mathrm{K}+: 4.02 \mathrm{mg} / \mathrm{dl}, \quad$ Urea: $17.38 \mathrm{mg} / \mathrm{dl}$, Creatinine: $0.93 \mathrm{mg} / \mathrm{dl}, \mathrm{Hb}: 13.5 \mathrm{gm} / \mathrm{dl}$;

Stool R/E:Normal;

HIV : Non Reactive;

Chest Xray: Normal; Anti-TPO:>600IU

During admission period the patient was treated with IV fluids and IV antibiotics, however his blood pressure was in lower range and was still feeling lethargic and anorexic with few episodes of vomiting on and off.

\section{On the 2nd day of admission -}

lab reports $-\mathrm{Na}: 137.0 \mathrm{mg} / \mathrm{dl}$, K:3.8mg/dl, Fasting Blood sugar:76mg/dl

USG abdomen: Normal, normal looking suprarenal glands;

TFT was in normal range.

8 AM serum cortisol - $<0.20 \mathrm{ug} / \mathrm{dl}(4.30-22.40 \mathrm{ug} / \mathrm{dl})$

serum ACTH was $1289.0 \mathrm{pg} / \mathrm{ml}(<10.0-46.0 \mathrm{pg} / \mathrm{ml})$; Mantoux test: Induration $12 \mathrm{~mm}$.

Patient was started on steroid replacement therapy based on low cortisol level and high ACTH level and his blood pressure increased, vomiting stopped and his appetite improved substantially and was discharged on thyroxine and steroid supplement therapy.

\section{Follow up examination:}

Two months after discharge patient was feeling reasonably well, he had gain $10 \mathrm{~kg}$ of weight, his skin was fairer than before. His BP was $120 / 70 \mathrm{mmHg}$ and his renal function tests were in normal range.

\section{DISCUSSION}

Hypothyroidism is a common condition. Laboratory confirmation of thyroid dysfunction is best achieved with serum free thyroxine and thyrotropin-secreting hormone assessments. So in this patient his Thyroid function Test 3 months back showed decreased Free T4 and Increased TSH and his Anti-TPO was strongly positive. As the most common cause of hypothyroidism is Hasimoto thyroiditis which is an autoimmune disease. Rarely, it may occur in combination with autoimmune failure of other endocrine glands (autoimmune polyendocrinopathy syndrome type 2, previously known as Schmidt's syndrome). ${ }^{16}$ In such cases, restoring normal thyroid function may precipitate adrenal failure. Clinicians should have a high index of suspicion for this condition in patients with Addison's disease, those with a family history of autoimmune endocrine gland failure, patients with one autoimmune endocrine disease who develop nonspecific or serious illness, and patients with type 1 diabetes mellitus whose insulin requirements drop without obvious explanation. The great danger in APS2 (as illustrated by this case) is treatment of a presenting hypothyroid state without recognition of concomitant hypoadrenalism. This may precipitate Addisonian crisis through two mechanisms. First, hypothyroidism reduces cortisol clearance. Second, hypothyroidism reduces the metabolic rate thereby reducing the need for cortisol. The increased metabolic rate accompanying thyroxine replacement increases the cortisol requirements that cannot be provided by the failing adrenals. The addition of thyroid hormone replacement increases cortisol clearance, thus decreasing circulating cortisol availability. Patients may die from ensuing Addisonian crisis. As in this case, our patient is a middle aged man of Brahmin cast with fair complexion who came to us with hyperpigmentation of skin, lethargy, anorexia and multiple episodes of vomiting and diarrhea. On admission his BP was very low and his fasting blood sugar was also in lower range, he was recently diagnosed as having overt hypothyroidism and was under thyroxine replacement since last 3 months. A high index of suspicion for adrenal failure should be maintained when patients with autoimmune thyroid disease or type 1 diabetes mellitus develop nonspecific but serious illness. Adrenal insufficiency is more difficult to document owing to the variable secretion of cortisol and broad range of normal values. The patient presented in this case had typical history of darkening of skin with lethary, anorexia and multiple episodes of diarrhea and vomiting which was more profound after thyroxine replacement therapy. After IV fluid resuscitation and IV antibiotics his blood pressure was still in lower range and his was still anorexic and not feeling well. This patient was diagnosed as having addison's disease as his lab investigations clearly showed that his $8 \mathrm{Am}$ serum cortisol level was very low and his serum ACTH level was remarkably high. If the clinical suspicion is high but cortisol levels are normal, an ACTH stimulation test provides the best assessment of adrenal function. As this patient had typical symptoms like lethargy, tiredness and anorexia with increased pigmentation of skin, his lab investigations clearly 
showed decreased cortisol and increased ACTH and during admission he had diarrhoea with multiple episodes of nausea and vomiting, his BP was very low and his fasting blood sugar was also in lower range and his symptoms were remarkably improved by steroid replacement therapy, although his serum electrolytes were in normal range we considered that he had addisonian crisis precipitated by thyroxine replacement therapy. As he had no history of PTB, no fever, his chest X-ray was normal, his mantoux test was negative and USG abdomen showed normal looking suprarenal glands, in the background of his autoimmune thyroiditis we considered that he had primary hypoadrenalism due to autoimmune adrenalitis as it is the most common cause. ${ }^{4}$ Both autoimmune thyroid disease and type 1 diabetes mellitus are common conditions. Rarely, one or both of these conditions is the presenting feature of potentially fatal auto-immune failure of several endocrine organs known as auto-immune polyendocrinopathy syndrome type 2 (APS2). Patients with adrenal failure may show multiple abnormalities of blood chemistry, but this patient had normal serum sodium and potassium level as hyponatremia is reported in only $90 \%$ of patients and hyperkalemia in $65 \%$ depending on the degree of destruction of the affected endocrine organs.

The management of APS2 is based on individualized, lifelong replacement therapy for the affected endocrine organs plus monitoring for development of insufficiency in other organs or the associated conditions. Patients must be monitored regularly with history, physical examination, and appropriate laboratory evaluation. Medi-alert and other measures should be taken to ensure that adrenal function is taken into consideration during illness, surgery, or emergency situations. Family members should also be made aware of the increased risk of endocrine disease, especially of APS2. The autoimmune destruction of several endocrine organs in APS2 results in clinical adrenal failure plus autoimmune thyroid disease or type 1 diabetes mellitus. Research indicates that autoimmune processes commonly attack multiple endocrine organs, although this may not result in sufficient damage to produce clinical symptoms. Physicians should be alert to the potential for additional endocrine conditions, particularly adrenal failure, in all patients with autoimmune endocrine diseases, especially those with insulin-dependent diabetes and autoimmune thyroid conditions.

\section{REFERENCES}

1. Addison T: On the Constitutional and Local Effects of Disease of the Supra-Renal Capsules, London, Highley, 1855.

2. Oelkers W: Adrenal insufficiency. N Engl J Med 1996; 335:1206-1212.

3. Arlt W, Allolio B: Adrenal insufficiency. Lancet 2003; 361:1881-1893.

4. Carey RM: The changing clinical spectrum of adrenal insufficiency. Ann Intern Med 1997; 127:1103-1105.

5. Schmidt MB. Eine biglanduloma Erkrankung (Nebennieren und Schilddrüse) bei Morbus Addisonii. Verh Dtsch Ges Pathol 1926;21:212-221.

6. Graber MA, Freed HA. Polyglandular autoimmune syndrome: A cause of multiple and sequential endocrine emergencies. Am J Emerg Med 1992;10:130 -132.

7. Betterle C, Dal Pra C, Greggio N, et al. Autoimmunity in isolated Addison's disease and in polyglandular autoimmune diseases type 1, 2, and 4. Ann Endocrinol 2001;62:193-201.

8. Riley WJ. Autoimmune polyglandular syndromes. Horm Res 1992;38: 9-15.

9. Khalil S, Evers ML. Case report:Autoimmune polyglandular syndrome. N J Med 1995;92:671- 674.

10. Heuss D, Engelhardt A, Göbel H, Neundörfer B (June 1995). "Myopathological findings in interstitial myositis in type II polyendocrine autoimmune syndrome (Schmidt's syndrome)". Neurol. Res. 17 (3): 233-7.

11. Greenspan, Francis S.; Gardner, David C. (2004). Basic clinical endocrinology. New York: McGraw-Hill. p. 103.

12. Falorni A, Laureti S, Santeusanio F. Autoantibodies in autoimmune polyendocrine syndrome type II. Endocrinol Metab Clin N Am 31: 369-389, 2002.

13. Vaidya B, Pearce S, Kendall-Taylor P. Recent advances in the molecular genetics of congenital and acquired primary adrenocortical failure. Clin Endocrinol 2000;53:403- 418.

14. Peterson P, Uibo R, Krohn KJ. Adrenal autoimmunity: Results and developments. Trends Endocrinol Metab 2000;11:285-290.

15. Davenport J, Kellerman C, Reiss D, et al. Addison's disease. Am Fam Physician 1991;43:1338 -1342.

16. Desmond A. Schatz, William E.Winter. Autoimmune polyglandular syndrome II: clinical syndrome and treatment. Endocrinol Metab Clin N Am 31: 339-352, 2002. 